\section{Iron Deficiency}

Good therapeutic results, together with other facts, have caused many investigators to regard these anaemic conditions as deficiency diseases. It is thought that an insufficient supply of iron may be of primary aetiological significance. This deficiency in iron is caused partly by food poor in iron, partly by decreased absorption of iron from food because of digestive disturbances. Woman, owing to menstruation, pregnancy, and lactation, has a larger expenditure of iron than man, and there has been a desire to explain the appearance of the disease predominantly in the female sex on this basis. Davidson and his associates, who have recently examined the iron content of the food of the poor people in Aberdeen, found that the amount in their diet was as a rule insufficient. In a series of 3,000 individuals from this element of the population they observed that a large number were anaemic, but that all of the cases with anaemia occurred among the women. About half of all the women were anaemic, and these anaemic individuals were, as a rule, between 18 and 50 years of age. In Sweden the poorer element of the population exists on a diet which is undoubtedly poor in iron (a small amount of meat, very little fruit, and practically no green vegetables); and it is possible that the numerous cases of carcinoma in women in our material may be related to this fact. A certain amount of support for this opinion is obtained from the fact that in Radiumhemmet's material of comparable cases of carcinoma among the better situated classes only 20 per cent. is made up of women, and of these women only two cases have had any sign of Plummer-Vinson's syndrome.

These aetiological speculations are, of course, indefinite. We must acknowledge that we do not yet know the cause of these anaemic conditions. If their aetiology were completely clear and we had, moreover, a truly specific therapeutic agent at our disposal, we should be able to speak of an effective prophylaxis in women against hypopharyngeal carcinoma in particular, and also against carcinoma of the mouth.

\section{Conclusion}

The syndromes discussed should command the attention of cancer investigators when looked at from this viewpoint, as these syndromes undoubtedly constitute excellent examples of conditions which are precancerous. Their tendency to predispose to carcinoma must be greater than, for example, that of syphilis, for a far greater proportion of our patients with carcinoma of the mouth and pharynx have these syndromes than have syphilitics, even though the incidence of syphilis probably is many times greater in the general population.

It also appears to be of great theoretical interest that in many of these cases the foundation for a carcinoma appearing at 50 or 60 years of age is already laid during the years immediately following puberty, or perhaps even earlier.

BiBLIOGRAPHY

Ahlbom, H. E.: Nord Med. Tidsskrift, 1936, xi, 171

Cameron, J. A. M.: Journ. Laryngol. and Otol., 1929, xliv, 168.

Davidson, L. S. P., Fullerton, H. W., Howie, J. W., Croll, J. M., Orr, J. B., and Godden, W.: British Medical Journal, 1933, i, 685 .

Davidson, L. S. P., Fullerton, H. W., and Campbell, R. M.: Ibid., 1935, ii, 195

Davies, D. T.: Lancet, 1931, ii, 385.

Faber, K.: Med. Klinik, 1909 , v, 1310

Idem: Berl. klin. Woch., 1913, i, 958.

IcGibbon, J.: Journ. Laryngol. and Otol., 1935, 1, 329

Kelly, B.: Ibid., 1919, xxxiv, 285.

Paterson, D. R.: Ibid., 1919, xxxiv, 289.

Idem: Proc. Roy. Soc. Med. (Sect. Laryngol.), 1931, xxiv, 1203.

Plummer: Quoted by Vinson.

Suzman, M. M.: Arch. Int. Med., 1933, li, 1.

Taylor, G. W.: New Ėngland Journ. Med., 1934, ccx, 1102.

Turner, A. L.: Journ. Laryngol. and Otoi., 1913, xxviii, $281 ; 1920$ xxxv, 34 .

Vinson, P. P.: Minnesota Med., 1922, v, 107.

Witts, L. J.: British Medical Journal, 1931, ii, 883.

\section{ACUTE INFECTIVE POLYNEURITIS * WITH A REPORT OF FIVE CASES}

BY

CHARLES PINCKNEY, M.B.CAMB., M.R.C.P.LOND. HONORARY PHYSICIAN TO OUT-PATIENTS, VICTORIA HOSPITAL FOR CHILDREN; HONORARY PHYSICIAN AND PHYSICIAN IN CHARGE, NEUROLOGICAL DEPARTMENT, KING GEORGE HOSPITAL, ILFORD; TEMPORARY ASSISTANT PHYSICIAN, ST. GEORGE'S HOSPITAL

Acute infective polyneuritis is a comparatively rare disease, appearing practically at any time, but mainly affecting young males between the ages of 20 and 40 . It is by no means a new disease, for Viets ${ }^{1}$ in his abstract records that Graves gave a clinical description of this disease in 1828, and Duménil in 1846 placed multiple neuritis as a definite entity.

The disease has been shown by Wilson ${ }^{2}$ to be caused by a living virus, which was successfully transmitted from man to monkey and recovered in pure culture; but in many later cases such conclusive evidence has not been obtained. A toxin, however, is produced which seems to have a selective action on the lower motor neurones, causing a chromatolysis of the ganglion cells of both the anterior horns and posterior roots, when a rapid segmental spread may occur, either ascending or descending to involve the whole cord. The toxin reaches the cord by absorption from the peripheral end-plates of the motor and mixed nerves, which show a true neuritis with swelling and fragmentation of their axis cylinders.

\section{Types of Cases}

Collier ${ }^{3}$ describes the cases as falling into two types. The first is that of a four-limb peripheral neuritis associated with an external ophthalmoplegia, the malady being apyrexial and painless with a rapid onset. In some of the cases the ophthalmoplegia is severe and may be absolute so far as the external muscles are concerned, the internal muscles escaping, while the limb paralysis may be slight, only showing itself by a weakness of the extensors of the periphery with an absence of reflexes. In other cases the limb paralysis is severe while the ophthalmoplegia is slight. The second group of cases resemble those described by Gordon Holmes ${ }^{4}$ and Bradford, Bashford, and Wilson ${ }^{2}$; they have a slight pyrexia with a less rapid onset. They also show a fourlimb polyneuritis, but accompanied by a facial paralysis and often a slight general bulbar weakness. Here again the limb paralysis is in some patients the dominating part of the picture, while in others the facial paralysis predominates.

A third group of cases, however, must be included which start as a lower-limb polyneuritis, spreading rapidly upwards to involve the trunk and finally the muscles of respiration. This group cannot be distinguished from what is known as Landry's paralysis, for there is little doubt that the two are due to a similar cause, and, as Grainger Stewart ${ }^{5}$ has stated, any distinction is artificial.

\section{Motor and Sensory Symptoms}

In all these groups the paralysed muscles are flaccid, but do not show much wasting, and the reflexes are sometimes surprisingly obtained when voluntary power is almost absent. The paralysis also need not be truly peripheral, and is often more proximal than distal, so that a patient may be seen to have some movements of his fingers and toes though unable to move arm or leg. Sensory symptoms are usually present, being described in the early stages as pain or numbness and tingling in

* Abstract of address to the Stratford Division of the British Medical Association, March, 1936. 
the limbs, later some impairment to light touch being commonly found. The sphincters are hardly ever involved, but an exception is seen in Case III. The electrical reactions tend to be sluggish, but do not show the reaction of degeneration which is found in poliomyelitis.

\section{CASE I}

A male, aged 30 , eleven days before admission to hospital, complained of headache and pains in the back, with nausea and vomiting. He had been playing tennis in the hot sun, and it was thought that he was suffering from the effect of this. Eight days before admission he complained of weakness in all four limbs, more marked in the legs than in the arms. On admission he was apyrexial, with a four-limb polyneuritis and slight right-sided facial weakness. The weakness of the upper arms was more marked than that of the forearms and fingers; there was some weakness of the muscles of the lower abdomen and back, with involvement of diaphragm ; contraction of the quadriceps was just obtainable, and the extensors and flexors of the foot were weak. All reflexes were absent. There was a slight loss of sensation to pin-prick and light touch tests in both legs. The electrical reactions were sluggish, but there was no reaction of degeneration. The cerebro-spinal fluid was examined on nine occasions; the colour varied from brown to yellow. There were never more than six cells present, and the proteins were always markedly increased, the highest reading being 0.25 per cent.

After four days the movements of the diaphragm became stronger, and after ten days the arms improved and the leg muscles were contracting well.

Gradual improvement was maintained for two months, when an acute exacerbation with rapid ascending spread of paralysis again occurred, involved the diaphragm, and was followed by bronchopneumonia and death.

\section{CASE II}

A male, aged 28 , one week before admission complained of sore throat; two days before admission complained of weakness of the left side of the face, with a feeling of numbness and difficulty in smiling, speaking, and talking; he had no vomiting. On admission there was slight pyrexia-99.5 $\mathrm{F}$.; ptosis of both eyes, complete in the left eye ; external ophthalmoplegia with nystagmus ; and bilateral facial paresis, more marked on the left than on the right. There was a four-limb polyneuritis, paralysis being more proximal than distal, so that movements of the fingers and toes were always possible ; all reflexes were absent. The sense of light. touch was impaired in the hands, arms, and legs. The cerebrospinal fluid was clear and yellowish, with never more than two cells per c.mm. ; protein was always markedly increased, up to 0.12 per cent.

After fourteen days the right facial weakness was improving and the abdominal reflexes were present; after three weeks he was able to write; after one month the knee-jerks were present; and after two months he was discharged with slight weakness of the left face only.

\section{CASE III}

A male, aged 44, nine days before admission complained of loss of power in the arms and legs, with retention of urine. On admission he was apyrexial and had a four-limb polyneuritis, with involvement of the trunk muscles and partial involvement of the diaphragm. There was no involvement of cranial nerves; weakness in the arms was more marked distally; the legs were completely paralysed; reflexes all absent, but there was no sensory involvement. Catheterization was necessary to relieve retention with distension of the bladder. There was no reaction of degeneration in the muscles. The cerebro-spinal fluid showed excess of albumin.

Eight days after admission slight movements of the fingers and toes were possible, and nearly full movements of the shoulders; the abdominal muscles also could be made to contract. After sixteen days the movements of hands and feet were much improved, but catheterization was still necessary to relieve the retention of urine. Ten weeks later he was discharged well.

\section{CASE IV}

A male, aged 27, five weeks before admission complained of weakness gradually spreading upwards in both legs, and later weakness of the arms, more marked in the hands and wrists. On admission he was apyrexial, and had a four-limb polyneuritis with involvement of the lower trunk muscles and the diaphragm; there was bilateral facial weakness with some bulbar involvement. The cerebro-spinal fluid was not examined.

Two days after admission there was complete diaphragmatic paralysis; soon after, however, improvement began. In ten weeks he was discharged to a convalescent home, able to walk with sticks. After three weeks there exacerbation occurred with a fresh spread of paralysis, so that on readmission he had a four-limb polyneuritis with complete paresis of the legs, partial paresis of the arms, and all reflexes abolished. One month later there was definite improvement, and after fourteen weeks he was discharged, walking with support.

\section{CASE V}

A male, aged 32 , nine days before admission had complained of coryza and sore throat; three days before admission ne complained of pins-and-needles in the arms and legs, with increasing weakness; two days before admission he developed nasal speech and diplopia. On admission he had vomited; had a four-limb polyneuritis, complete external ophthalmoplegia of both eyes, and the pupils reacted sluggishly to light and accommodation. The trunk was not involved, and movements of the hands were obtainable. There was complete paresis of the legs, and the reflexes were absent in both arms and legs. The cerebro-spinal fluid showed no increase in cells, but a marked excess of protein.

After ten days the palatal muscles recovered and the ophthalmoplegia began to improve. Six weeks later he was able to walk and the knee-jerks were obtained. After two months he was discharged well, though there was still some diplopia present.

\section{Discussion}

If an attempt is made to fit these cases into the above three clinical groups we find that Case I, at its onset, and Case IV seem to fall into the second group. But Case I terminated fatally with a rapid ascending spread of paralysis identical with that of the cases forming the third group. Cases II and V seem to combine the characteristics of the second and third groups, for Case II showed both an ophthalmoplegia and a facial paralysis, while Case V showed an ophthalmoplegia with a bulbar paresis. Case III is of interest in showing sphincter involvement with no affection of the cranial nerves. From this it is apparent that difficulty will occur when trying to fit cases into any definite clinical groups, and that any such grouping must be artificial.

\section{Prognosis and Treatment}

The mortality is high, but this varies in the series of cases which have been described, tending to be higher in the small epidemics of this disease. In Bradford's series eight died out of thirty, while in the present series out of five patients one died. The prognosis must always remain, however, somewhat uncertain, even later in the course of the disease, owing to the frequency with which exacerbations, with a further rapid increase of paralysis, may arise, but the cases which recover tend to do so completely. I have been tempted to give a prognosis based on the appearance and chemistry of the cerebrospinal fluid, a brown or yellowish tinged fluid with a high protein content, which points to a vigorous reaction, appearing to justify a better prognosis than a clear fluid which shows only slight or no alteration.

There is no specific treatment, but frequent lumbar punctures, especially where a high protein fluid is obtained, continued until that protein content falls, appears to hasten the rate of recovery. 


\section{Conclusions}

1. Five cases are described of acute infective polyneuritis, a disease which appears to have become more prominent recently.

2. The three main clinical groups usually described are recorded.

3. The difficulty of accepting any such definite grouping is discussed.

4. A prognosis based on the appearance and chemical findings of the cerebro-spinal fluid is suggested.

I am indebted to Dr. Anthony Feiling and Dr. Harold Pritchard, under whose care three of the patients were admitted, for permission to publish these cases.

\section{REFERENCES}

Viets, H. B.: Arch. Neurol. and Psychiat., 1934, xxxii, 377. Bradford, J. R. Bashford, E. F., and Wilson, J. A.: Quart. Journ. Med., 1918-19, xii, 88.

Collier, James: Morison Lectures, Edinburgh Med. Journ., 1932 xxxix, No. 10

Holmes, Gordon: British Medical Journal, 1917, ii, 37.

Stewart, T. Grainger: Ibid., 1925, ii, 461.

\section{Clinical Memoranda}

\section{An Unusual Case of Mastoiditis}

The following case seems to be of interest as an unusual and puzzling type of mastoid disease.

A boy aged 5 was admitted to hospital on November 23rd, 1935, having been taken ill four days previously, and since complained of almost continuous severe frontal headache (chiefly right-sided), with frequent vomiting. He had a sore throat a week previously, and frequent earache (without otorrhoea) for six months; the earache had recurred on the right side during the last two days. Tonsils and adenoids had been removed two years previously.

On admission his temperature was $99^{\circ} \mathrm{F}$.; he had a coated tongue, flushed cheeks, and slight circumoral pallor. Pharyngitis was present; the left ear drum appeared normal, and the right oedematous and dull; no glands were felt in the neck. No abnormality was found in the nervous system. The nasal edges of the optic disks were a little blurred.

On November 25th his temperature rose to $102^{\circ}$; the posterior quadrant of the right drum was red and bulging, and there was a large very tender gland in the right posterior triangle of the neck. Myringotomy was performed on the right side, and a little serous exudate found, but no pus. During the next week the drum was obscured by inflammatory oedema of the posterior meatal wall, which was attributed to trauma during myringotomy ; when this subsided the drum was found to appear healthy. A transitory injection of the left drum was observed. At no time was there any oedema or tenderness over either mastoid. $X$-rays taken on November 29th and December 5th showed dense mastoids, with no difference between the two sides and no evidence of infection. Swabs taken from the throat and right external auditory meatus on November 27th, and from the throat on December 5th, gave on culture profuse growths of Staphylococcus aureus.

Headache and earache seemed to diminish from the time of admission, and neither was complained of after November 28th. From November 25th to December 11th the temperature varied irregularly between $98^{\circ}$ and $103^{\circ}$. The boy was irritable at times, but at no time unduly drowsy ; he remained interested in his surroundings and in comic papers, and anxious for meals, and did not vomit. On November 28th mild papilloedema was definitely present ; this gradually subsided (more completely in the right eye than the left) for a week, and then increased until operation. Kernig's sign was positive on November 30th for the first time, and neck rigidity remained, although the tender swelling in the neck was subsiding. Rigidity persisted in varying degrees until after operation. No other nervous signs were observed, except diminution of the left knee-jerk on December 4 th and subsequent days.
Results of lumbar puncture are shown in the following table:

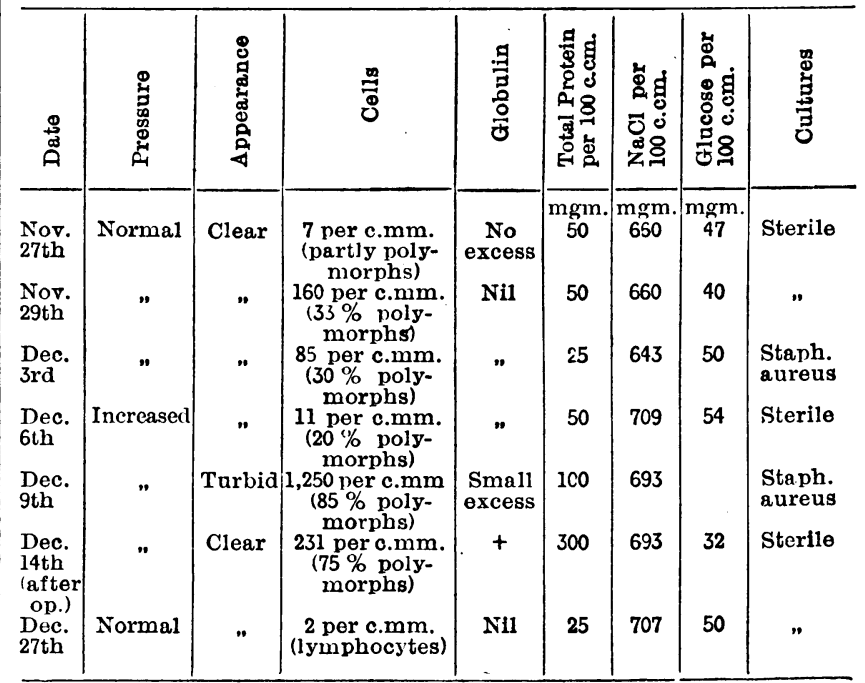

Blood counts showed a moderate polymorphonuclear leucocytosis; blood culture was negative.

On December 11th, because of the history of right-sided otitis media, and in spite of the absence of signs of mastoiditis, it was decided to operate on the right mastoid. Pus was found in the mastoid antrum and such cells as were present.

There was a large peri-sinus abscess, and the lateral sinus was thrombosed from the torcula to the jugular bulb. The lateral sinus having been opened up and packed, an incision was made in the neck over the jugular vein. The latter was found, with difficulty, embedded in a mass of glands, and was filled with organizing clot down to and beyond the thoracic outlet. The thrombus in the neck was left in situ. a drainage tube being inserted into the wound in the vein.

The temperature was settled within a week of operation " the patient made an uneventful recovery, and thoroughly enjoyed the Christmas festivities.

I am indebted to Dr. O. C. M. Davis and to Mr. AngellJames, who performed the operation, for permission to publish this case.

M. C. A. Robson, B.Ch., M.R.C.S. Bristol Children's Hospital.

\section{Multiple Cardiac and Pericardial Ruptures}

The following record is of a rare and interesting case.

On the evening of May 8th, 1936, I was called in to a case of sudden collapse, a man aged 67 ; unfortunately he was dead within a few minutes, and as he had not been seen recently by any medical man I was asked to perform a post-mortem examination The pericardial sac was full of blood. There was free fluid in the mediastinum, and there were three large ruptures in the posterior wall of the pericardium. The whole of the anterior wall of the pericardium was calcified-in parts a quarter of an inch thick-and the posterior wall was slightly calcified. The right auricle and the first part of the aorta, which was very atheromatous, were also ruptured. The lungs and pericardium were very adherent, as were the meninges. The right kidney was almost nonexistent, and the left kidney was twice normal size. The heart was not enlarged and the muscle was in fair condition.

I have had great difficulty in obtaining any history. Prior to his death the patient had just bent down and said he felt giddy; his previous medical history was a recent urethral stricture. The lack of previous history is quite in keeping with these cases. The main interest lies in the multiplicity of the ruptures and in the fact that there are only about 100 recorded cases.

Gillingham, Kent.

Dalton Y. Carter. 